\title{
Availability analysis of railway track circuit
}

\section{A P Patra* and U Kumar}

Luleå Railway Research Center, Division of Operation and Maintenance Engineering, Luleå University of Technology, Sweden

\begin{abstract}
Railways are expected to operate with ever increasing availability. The availability of railway systems and subsystems influences the overall operational availability. As the track circuit is a key component of railway signalling and control, it can contribute significantly to the loss of availability of the railway system. One way to increase the availability of the track circuit is through implementing proper a maintenance policy. In this article, Petri-Nets have been used to develop models for availability analysis of the track circuit for both time-based and condition based maintenance. A sensitivity analysis has been performed to estimate the effect of maintenance parameters on availability performance using cost-benefit analysis.
\end{abstract}

Keywords: Track circuit, Availability, Petri-Nets

\section{INTRODUCTION}

The railway track circuit is an electrical device installed on the track to provide an indication of train presence on a particular section of a track. The track circuit has been the most commonly used train detection device besides the axle counter. The advantage of the track circuit over the axle counter is its ability to detect rail breaks. Its correct operation is critical to obtaining dependable train operations in most main line and metro systems. The track circuit is a fail-safe device ensuring that any fault results in the signal light turning red, and in trains being prevented from entering the associated track section. However, this fail-safe property also has the potential to cause significant train delays if the system becomes unreliable. Any track circuit failure can cause significant disruption to rail services and hence can become a safety risk due to delay in the restoration of normal service. Over 12,000 track circuit failures were reported in the UK during 2004-2005, resulting in 1.5 million minutes of attributable delay. Typically, the UK railway infrastructure manager can be penalised with a penalty of $£ 20-60$ per delay minute arising from infrastructure failure [1]. Therefore, the ability to detect and diagnose track circuit failures in order to provide a fast response to failures/incidents has significant economic benefits.

Reduction of the un-availability caused by track circuit failures must be accomplished using an assured methodology. This methodology usually requires either an optimisation or a complete overhaul of the maintenance processes in place. This starts with understanding the causes of track circuit failures, which can either be within the track circuit itself, e.g. component or systemic failure, or result from a failure of part of the infrastructure, such as a poor track bed or related components. Railways all over the world follow a range of different track circuit maintenance policies. However, the problem is to find the cost-effective 
maintenance policy which will provide higher availability of the track circuit at a lower life cycle cost. To address this problem, it is important to determine the life cycle of the track circuit and the relationships between the failure distribution and the maintenance policy. The cost of each maintenance policy can be calculated by adding the direct maintenance costs and the consequential costs, e.g. the penalties due to train delay. This article provides a comparison between the track circuit availability achieved using scheduled maintenance and that achieved using condition based maintenance. The influence of the maintenance parameters on the availability in both cases is studied for track circuits currently in use across Sweden. There are different types of track circuits being used by infrastructure managers, e.g. DC track circuits, AC track circuits and audio frequency track circuits. In this article our comparisons have concerned the maintenance strategy for the DC track circuit which is used by the Swedish National Rail Administration (Banverket).

The DC track circuit is the simplest and least costly type of track circuit used for train detection. Fig. 1 presents the schematic diagram of a DC track circuit.

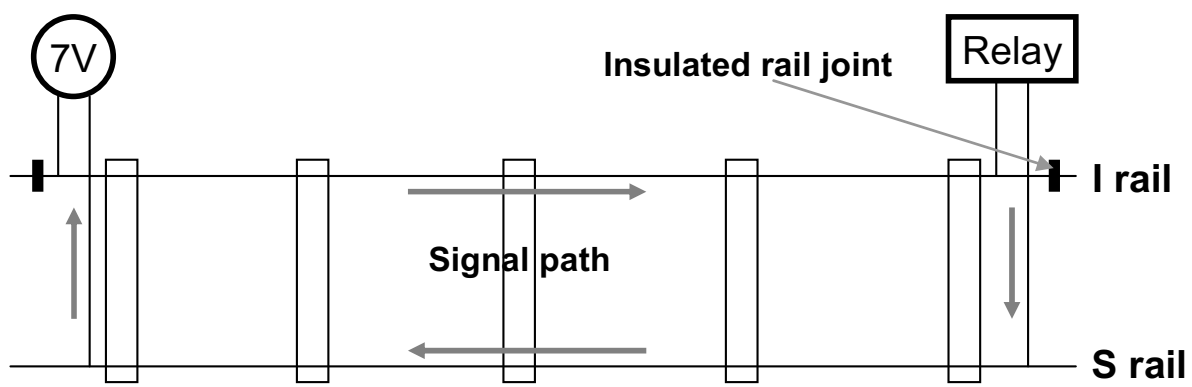

Fig. 1 Schematic diagram of DC track circuit

Banverket's the train detection system uses the two rails on a track, the common rail (S-rail) and the information rail (I-rail), to locate the position of the trains. The S-rail is continuous and is grounded and used for the track return current. The I-rail, on the other hand, has gaps with an insulated rail joint between the different sections of the rail. The polarity of each section is reversed so as to prevent the supply to one circuit from powering the adjacent circuit. The gaps isolate each section of the I-rail with a different potential. When no trains are on the line, there is a negative or positive potential between the rails. When a train moves into a new section (over a gap to a new rail section on the I-rail), the wheels complete the circuit connection and the potential drops to zero. The energized relay for the occupied section holds the voltage drops indicating where the train is on the track [2]. This track circuit design is failsafe; i.e. any failure in the track circuit turns the signal to red. Thus, the safety of the track is not compromised. However, frequent track circuits failures affect the reliability and availability of the track.

To achieve a reliable system, the factors which could influence the reliability of the system need to be identified, their effects need to be assessed and the causes of these effects need to be managed throughout the life cycle of the system. Railway system reliability is influenced in three ways [3]: 
- System conditions: the sources of failures are introduced internally within the system at any phase of the railway system life cycle. These failures are incurred by the design and manufacturing of the components or the system.

- Operating conditions: the sources of failures result from the operating system methodology. These failures are also incurred by environmental conditions.

- Maintenance conditions: the sources of failures are caused by maintenance actions.

The functionality of a track circuit is affected by the failure of its components, by changes in track characteristics or by track maintenance. The DC track circuit failure modes include:

- The failure of track circuit components

- Insulated rail joint failure

- Rail breaks

- Lower ballast resistance

- False alarms

Faults in track circuit components can be due to power supply failure or failure of the relay. There can be loose cable connections due to vibrations and cable discontinuities due to breaks in the cable caused by track maintenance actions, e.g. tamping. These failures are intermittent and random in nature. The failure distributions of such events are likely to follow exponential probability distributions. Insulated rail joints have shorter service lives than most other track components. The frequency of insulated joint failures is influenced by the relative and continuous weight (tonnage) of the traffic using the rails. Therefore, the frequency of failures of insulated joints is high in heavy haul. Capturing condition information about joints and taking preventive action remain challenges, as it is still proving difficult to obtain reliable trending information on the condition of joints. The failures of these joints are timedependant and increase with time and generally follow Weibull probability distributions. Consequently, time-based inspection is recommended as an approach to detecting degradations in insulated joints and replacing them before they fail. However, it is not easy to optimise the inspection periods in a track section with a number of insulated joints installed at different times. Broken rails follow similar probability distributions to those of insulated joint failures.

The track circuit operates at a specified electrical ballast resistance. When the electrical resistance of the ballast is lower than the specified value, the current flow along the rails drops and de-energizes the relay, which makes the track circuit non-functional. This is a frequent occurrence as a result of wet or dirty ballast. Ballast cleaning is necessary to keep the ballast dry and the electrical resistance above the minimum value. Occurrences of these kinds of failures are intermittent in nature and likely to occur more during winter than in summer. False alarms where the track circuit equipment has triggered a fault but where no fault is found are phenomena which are registered as NFF. These faults occur with no prior notification and are unpredictable in nature. False alarms normally are a significant subset of track circuit failures. As track circuit failures stop the rail service operation, it is necessary to detect degradation in track circuits and repair them before they actually fail. The following sections describe the features of scheduled maintenance and condition based maintenance, the differences between the two types of maintenance, and the benefits of condition monitoring over scheduled maintenance. 


\section{MAINTENANCE POLICY}

Infrastructure managers generally employ a scheduled (time based) maintenance regime for track circuits. The objective of this maintenance is to detect any degradation in the track circuit regularly and perform preventive maintenance to bring it back to a known acceptable state. This type of maintenance regime is expensive and time-consuming, since inspection needs to be carried out on every track circuit periodically (e.g. every 6 weeks for DC track circuits, which is the interval used by Banverket). However, sudden failures can, and do, occur between scheduled maintenance actions. In the event of such failures, urgent trackside corrective maintenance is carried out, which is costly, particularly when it has to be carried out during traffic hours. The periodic inspection of track circuits consists of measuring the voltage and current at different locations of track circuits, checking the insulated joints, inspecting the ballast condition, etc., to capture degradations (if any). If the measured current and voltage are different from the specified value, the track circuit is degraded and maintenance is carried out. However, if the occurrences of degradations of track circuits are random in nature, then it is difficult to optimise the scheduled maintenance interval in order to increase the system availability and reduce the cost.

Condition monitoring systems are designed and implemented so as to learn about system degradation and therefore reduce the maintenance burden and cost. The ability to detect degradation, identify certain incipient faults and/or provide diagnoses of failed track circuits, in a more 'intelligent' way, would have significant operational and economic advantages. One of the most important aspects of a robust track circuit condition monitoring system is its ability to identify degradation and failure modes, then to detect and locate a fault when it occurs, and to predict incipient failures so that potential damage can be avoided. Preventive maintenance can be performed before total failure [4]. The potential benefits of track circuit condition monitoring include:

- Improvement in the track circuit reliability and availability - by reducing the downtime during operational hours and hence reducing train delays.

- Improvement in the post-incident analysis - by improving the fault diagnosis capabilities, in particular by locating and classifying faults in a failed track circuit.

- Reduction in the number of 'Tested OK on arrival' events.

- Reduction in the scheduled down time - periodic routine maintenance tasks on the track circuit may not be required.

- Reduction in the safety risk associated with maintenance activities — by reducing the amount and length of trackside work.

- Reduction in the maintenance and replacement costs - the track circuit units can be maintained or replaced just-in-time if predictive condition monitoring can be achieved.

- Overall improvements in the track circuit performance - to provide a better understanding of the signalling network, optimise the train control system, reduce the penalty costs and increase the quality of rail services.

These are the potential advantages of track circuit condition monitoring systems. However, balanced against these advantages are the costs of establishing the requirements, of procuring, installing, "learning" and adapting the system and also of managing its own reliability. Therefore, the ability of the system to detect the probability of faults is a combination of the detection probability of the faults to be detected by the monitoring system and the reliability of the monitoring system itself. 
In this paper we have modelled availability as a factor of the detectability of the monitoring system. The decision to adopt either scheduled maintenance or condition based maintenance is taken based on an evaluation of the cost-benefit analysis of both types of maintenance philosophy

\section{AVAILABILITY MODELLING}

Track circuit availability modelling has been performed by using Petri-Nets [5]. A standard Petri-Net consists of a set of places, a set of transitions and a set of directed arcs. Directed arcs connect places to transitions and vice versa. The places of Petri-Net model represent the degrading states of the track circuit and the transitions represent the time delay between the two degradation states. The modelling has been supported by the software tool GRIF. The following sections describe how estimates of availability for the two approaches to maintenance have been made.

\subsection{Residual life}

As discussed in the previous section, track circuit degradations and failures can occur for different reasons. A framework is presented in Fig. 2 for the treatment degradation and failure data of track circuits in order to calculate the reliability parameters. These failures constitute all the failure modes of the track circuit. As illustrated in the figure, in the present scenario track circuits are inspected at scheduled time intervals to check them for any degradation. If the track circuit is degraded, this is treated as a condition failure and preventive maintenance is carried out on the track circuit to bring the track circuit to good state. The track circuit reaches a degradation state in between two inspections, but this state can only be detected and the track circuit can only be maintained during the next inspection. In order to estimate the effect of the inspection interval on detecting a degradation of the track circuit before its functional failure, we need to calculate the time to degradation (degradation life) and the time from degradation to functional failure (residual life) of the track circuit.

Fig. 2 serves as an example of the representation of real data for the maintenance of different track circuits (TC1, TC2, etc.) in relation to time. During inspection, if a track circuit is found to be degraded, the degradation is termed as conditional failure and preventive maintenance is carried out. If the track circuit goes out of order, i.e. functional failure occurs, corrective maintenance is carried out. It is assumed in this paper that both these maintenance actions bring the track circuit to an 'as-good-as-new' state. It is also assumed in the paper that any manual inspection that is carried out on the track circuit at a scheduled time interval is perfect and free from any human error. This means that, if a track circuit is degraded, it is always detected during the inspection. It can be said that, when a track circuit is maintained preventively or correctively, the degradation must have occurred between the maintenance point of time and the last inspection point of time. This is shown as the occurrence of a degradation zone in the figure.

As the point of occurrence of the degradation between two inspections or between the functional failure and the last inspection is uncertain, the probability distribution of the degradation life can be estimated considering the interval data for degradation. Once we obtain the degradation life distribution, the next step is to estimate the residual life. The residual life is the remaining life in a track circuit from the point of degradation until it 
experiences a functional failure. When estimating the residual life of the track circuit, we should also consider undetected degradation times, which are also termed as suspended residual life.

A model has been developed in Petri-Nets to calculate the undetected degradation time (see Fig. 3). State 1 denotes that the track circuit is in good state and state 2 that it is in degraded state. Degradation of the track circuit brings the track circuit from state 1 to state 2 . State 3 and 4 denote the inspection states. Inspection is carried out at a scheduled interval. A degraded track circuit remains in a degraded state until the next inspection commences. For an inspection interval of 1000 hours, by simulating the Petri-Net models we can see that the undetected degradation time is half of the inspection interval, i.e. 500 hours (see Fig. 4).

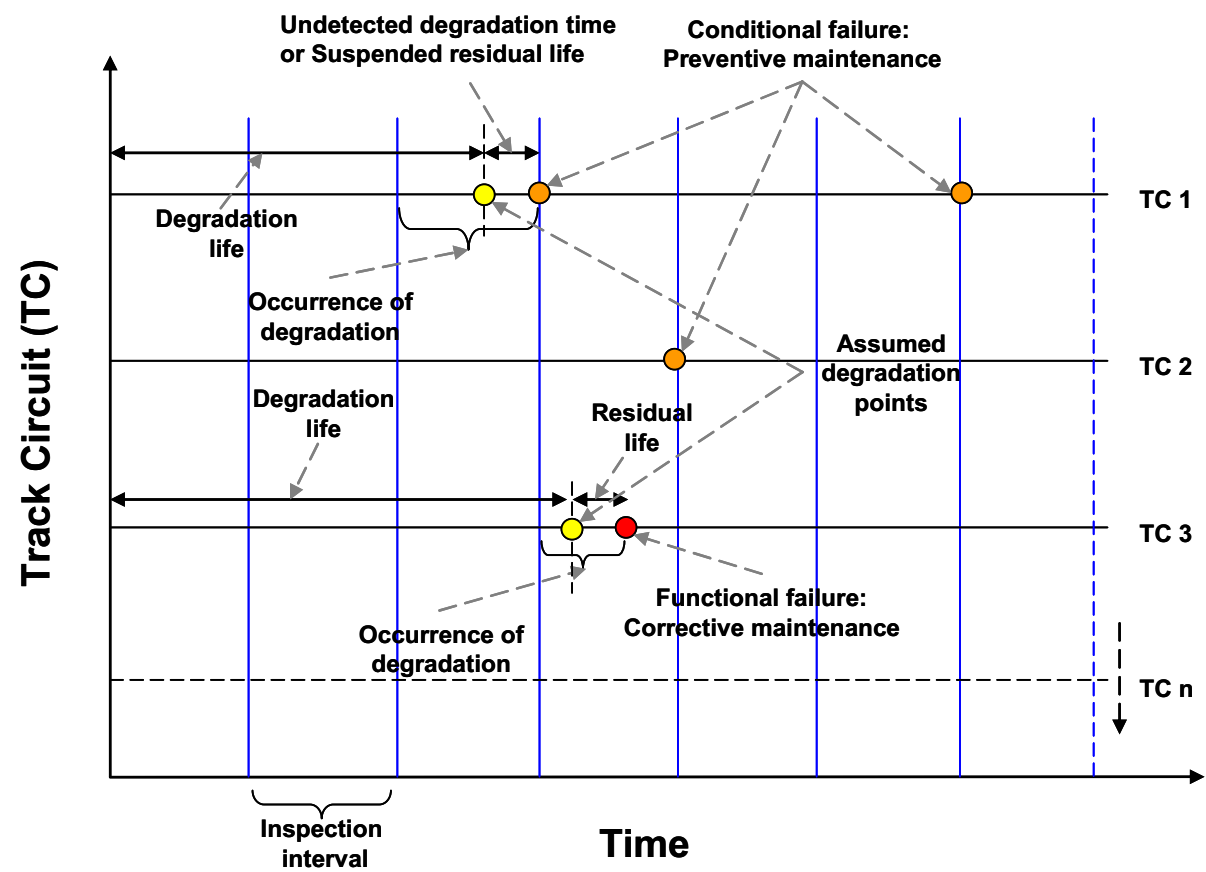

Fig. 2 An example of data representation for track circuit degradations and failures

The probability distribution of the degradation is assumed to be a Weibull distribution. It was seen that for other distributions, the undetected degradation time also remains half of the inspection interval. This result can also be inferred for the residual life calculation; i.e. the residual life is half of the time between the functional failure and the last inspection. Considering different values for the residual life and the suspended residual life (undetected degradation time), we can estimate the probability density function for the residual life. 

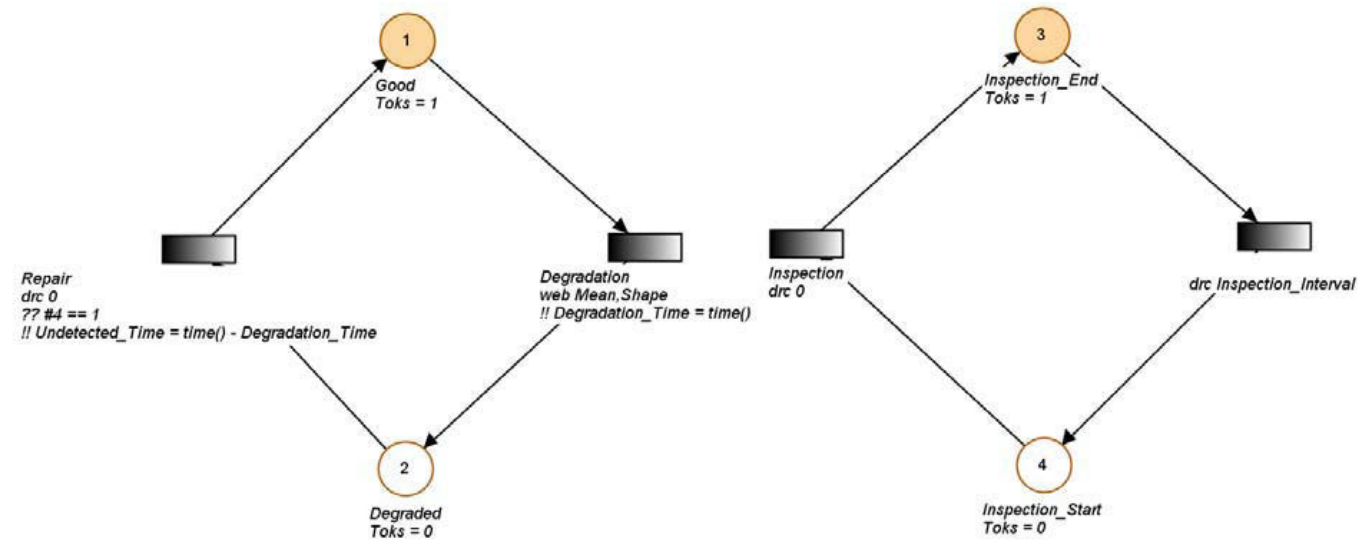

Fig. 3 Petri-Net model for estimation of undetected degradation time

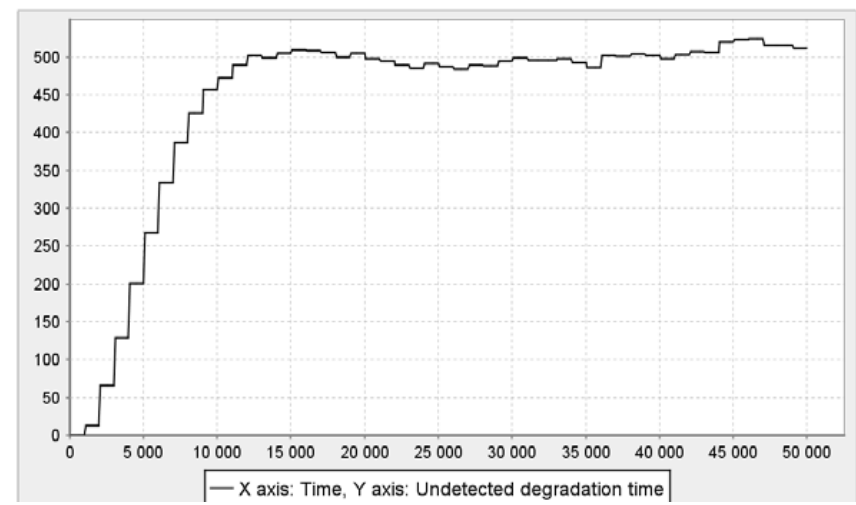

Fig. 4 Undetected degradation time

\subsection{Scheduled maintenance}

The availability estimation for the track circuit only considers the corrective maintenance. It does not consider the preventive maintenance, as the preventive maintenance is carried out during train-free periods; i.e. i.e. the unavailability during non-traffic hours is not included. A Petri-Net model for estimating the availability with the scheduled maintenance policy is shown in Fig. 5. State 1 denotes that the track circuit is in good state. The track circuit goes to state 2 when degradation occurs. It further goes to state 3 (failed state) depending upon the probability distribution of the residual life. Corrective maintenance in state 3 brings the track circuit back to good state. States 4, 5 and 6 are the inspection states. Inspection is carried out at every inspection interval. After the inspection is carried out, if it is found that the track circuit is in degraded state (state 2), preventive maintenance is performed on the track circuit and the track circuit goes back to good state.

For example, for each track circuit, the degradation follows a Weibull distribution with scale parameter 5000 hours and shape parameter 1.8, and the residual life distribution is a Weibull distribution whose scale and shape parameters are 700 hours and 1.2 respectively. The mean 
down time for both preventive and corrective maintenance is 2 hours and the inspection time is 0.5 hours. For an inspection interval of 1000 hours, the availability of the track circuit with respect to time is shown in Fig. 6. As shown in the figure, we obtain a steady state availability of 0.99985 for each track circuit. For a typical track section of $300 \mathrm{~km}$, we can have an average of 200 track circuits. If we need to calculate the availability of the section due to track circuits, we must consider the delay aspects of track circuits due to their unavailability. Different indicators of availability are illustrated in [6]. In this article the authors define the availability of a section of track as the probability that any failure in that track section will not induce any delay to the trains. Based on this definition, the availability of the track section is given by:

$$
\mathrm{A}_{\mathrm{s}}=1-\left[\left(1-\mathrm{A}^{\mathrm{n}}\right) * \mathrm{P}_{\mathrm{d}}\right]
$$

$\mathrm{n}=$ Number of track circuits in the section

$\mathrm{A}=$ Availability of each track circuit

$\mathrm{P}_{\mathrm{d}}=$ Probability of delay

To explain how this expression is developed, let us consider the simple case of three track circuits in a track section. A is the availability and $\overline{\mathrm{A}}$ is the un-availability of each track circuit i.e. all the track circuits are considered to be identical.

The probability of at least one of the three track circuits being un-available is given by:

$$
\overline{\mathrm{A}}_{3}=\overline{\mathrm{A}}+\overline{\mathrm{A}}+\overline{\mathrm{A}}-\left(\overline{\mathrm{A}}^{*} \overline{\mathrm{A}}\right)-\left(\overline{\mathrm{A}}^{*} \overline{\mathrm{A}}\right)-\left(\overline{\mathrm{A}}^{*} \overline{\mathrm{A}}\right)+\left(\overline{\mathrm{A}}^{*} \overline{\mathrm{A}} * \overline{\mathrm{A}}\right)
$$

The un-availability of the track circuits is independent but not mutually exclusive (e.g. $\mathrm{P}(\mathrm{A}+$ $\mathrm{B}+\mathrm{C})=\mathrm{P}(\mathrm{A})+\mathrm{P}(\mathrm{B})+\mathrm{P}(\mathrm{C})-\mathrm{P}(\mathrm{A}) * \mathrm{P}(\mathrm{B})-\mathrm{P}(\mathrm{B}) * \mathrm{P}(\mathrm{C})-\mathrm{P}(\mathrm{C}) * \mathrm{P}(\mathrm{A})+\mathrm{P}(\mathrm{A}) * \mathrm{P}(\mathrm{B}) * \mathrm{P}(\mathrm{C}))$

Further, the probability of at least one of the three track circuits being un-available is also equal to one minus the joint probability that none of the track circuits are un-available.

Thus, $\bar{A} 3=1-\left[(1-\overline{\mathrm{A}})^{*}(1-\overline{\mathrm{A}})^{*}(1-\overline{\mathrm{A}})\right]=1-\mathrm{A}^{3}$

If $P_{d}$ is the probability of delay, then the un-availability of the track section is given by (1$\left.\mathrm{A}^{3}\right) * \mathrm{P}_{\mathrm{d}}$.

Similarly, for $n$ track circuits, the un-availability of track section will be $\left(1-\mathrm{A}^{\mathrm{n}}\right) * \mathrm{P}_{\mathrm{d}}$.

Hence, the availability of the track section is $\mathrm{A}_{\mathrm{s}}=1-\left[\left(1-\mathrm{A}^{\mathrm{n}}\right)^{*} \mathrm{P}_{\mathrm{d}}\right]$

If $P_{d}$ is 1 (i.e. any un-availability of a track circuit in a section induces a certain delay), then $A_{s}=A^{n}$, where as, if $P_{d}$ is 0 (i.e. there is no train delay in the case of any un-availability of a track circuit), then $A_{s}=1$.

In this article it is assumed that any track circuit failure in a track section will induce train delay, and therefore the availability of the track section $=\mathrm{A}^{200}=0.99985^{200}=0.9704$. 


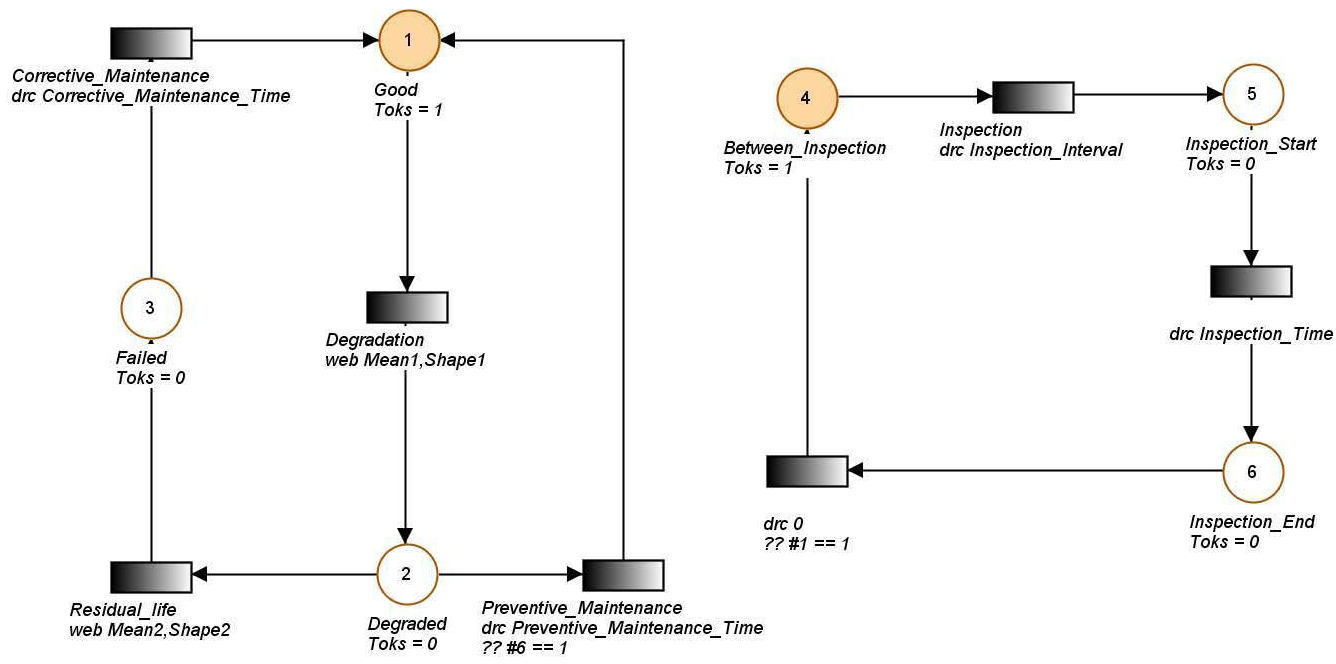

Fig. 5 Petri-Net model for estimation of availability for scheduled maintenance

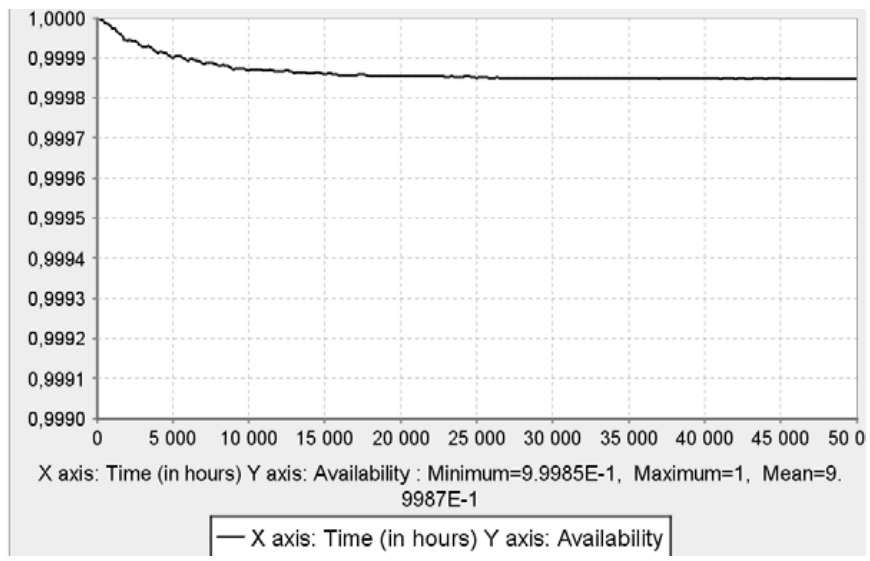

Fig. 6 Availability of track circuit for scheduled maintenance interval 1000 hours

If we want to increase the system availability, we need to perform a sensitivity analysis on the inspection interval. Fig. 7 illustrates the change in the section availability of a track circuit with a change in the inspection interval. If we want to optimise the inspection interval in terms of cost effectiveness, we need to calculate the cost of inspection as well as the cost of delay as described earlier. 


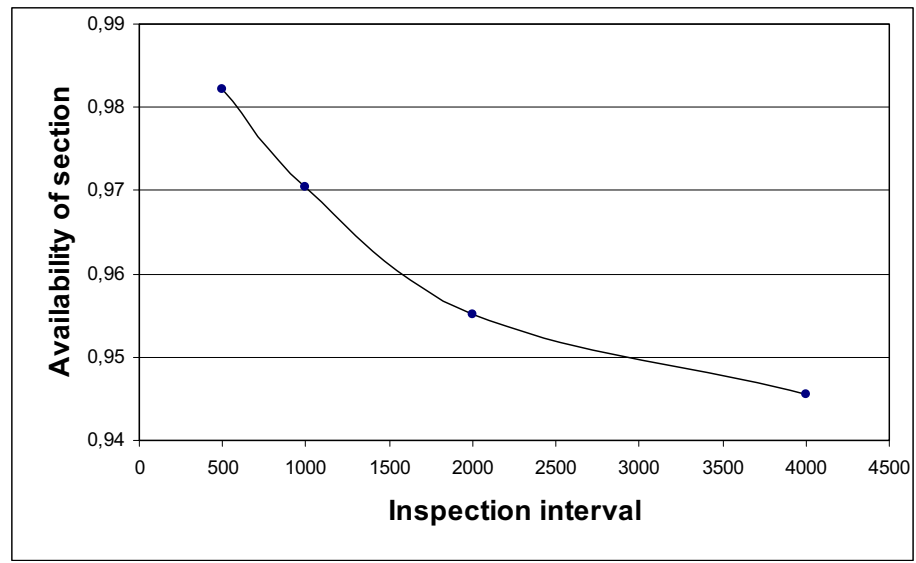

Fig. 7 Section availability vs. inspection interval

\subsection{Condition based maintenance}

As discussed earlier, condition monitoring on the track circuit will increase the availability of the track circuit. In this section we will illustrate the proportional increase in the availability of the same track circuit if we change the maintenance policy from scheduled maintenance to condition based maintenance. A Petri-Net model for estimating the availability with a condition based maintenance policy is shown in Fig.8. The detection probability of the condition monitoring system is considered in the model. As shown in the figure, when the track circuit is in degradation state, it can either go to detected degradation state (state 3 ) or un-detected degradation state (state 4), depending on the detection probability.

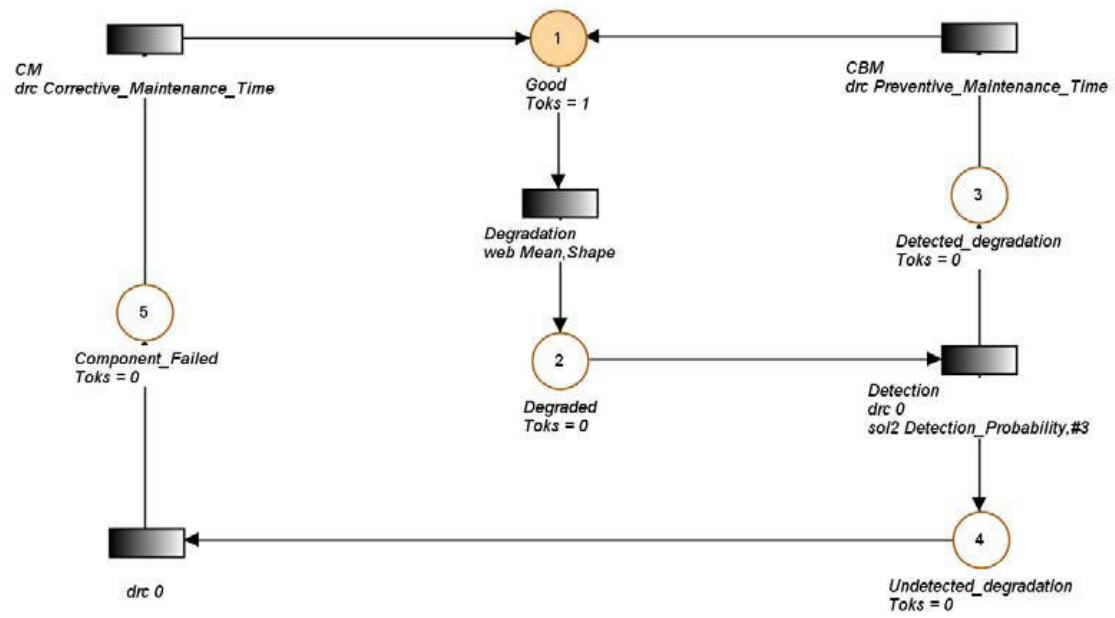

Fig. 8 Petri-Net model for estimation of availability for condition based maintenance

In this model it is assumed that the condition monitoring system detects the degradation in the track circuit all over its residual life. Therefore, the degradation life distribution in this case is 
the combination of the degradation life and the residual life distribution in the earlier example. If we consider the same failure and maintenance parameters as those considered for scheduled maintenance and perform a sensitivity analysis of the section availability with respect to the detection probability, we see the result in Fig. 9.

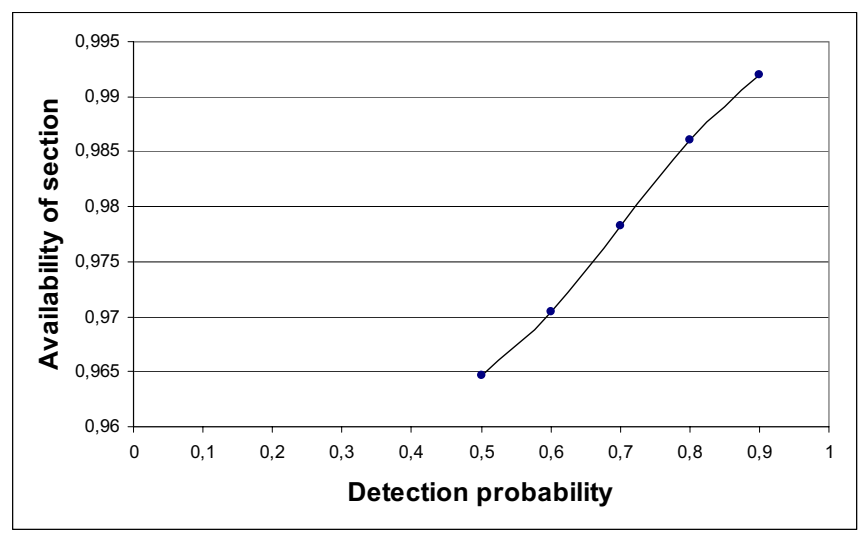

Fig. 9 Section availability vs. detection probability

As shown in the Fig. 9, one can achieve the same availability of the track section as achieved using current practice (scheduled maintenance with an interval of 1000 hours) by incorporating condition monitoring devices which have a detection probability of only $60 \%$. However, it is very likely that condition monitoring systems have a higher detection probability than $60 \%$. With a higher detection probability, we can achieve higher availability. However, to compare the cost effectiveness of condition based maintenance with that of scheduled maintenance, we must perform a cost-benefit analysis of each maintenance policy.

\section{COST-BENEFIT ANALYSIS}

As decisions on maintenance policies have a major impact on the operations of the system, the cost-effectiveness of long term design and maintenance decisions should be guaranteed. Cost-benefit analysis, an engineering economics technique, can be utilised to focus on maintenance strategies to minimise the maintenance cost in the system's life cycle, while meeting the dependability requirements. When comparing maintenance policies, the maintenance cost should include consequential costs (e.g. penalties due to train delays) in addition to direct maintenance costs. In the case of the scheduled maintenance policy, the maintenance costs include labour costs and equipment costs for inspection. If we want to change the maintenance policy to a condition based policy, we need to include the acquisition costs of monitoring systems, as well as the validation time and the costs for proving and developing the applicability of these systems and finally the cost of maintenance (i.e. corrective replacements) of the monitoring systems themselves. In both the cases we need to estimate the corresponding train delay and calculate the penalties.

Train delay consists of two types of delay, i.e. primary delay and secondary delay. Primary delay is due to some external circumstances, whereas secondary delay is caused by other trains. As secondary delay depends on the infrastructure design and train time schedule, in this paper we consider only primary delay. Failures of track circuits also directly affect the 
primary delay of trains. Fig 10 illustrates the primary delay incurred to trains because of track circuit failure. It is assumed that the headway between the trains is big enough not to induce any secondary delay.

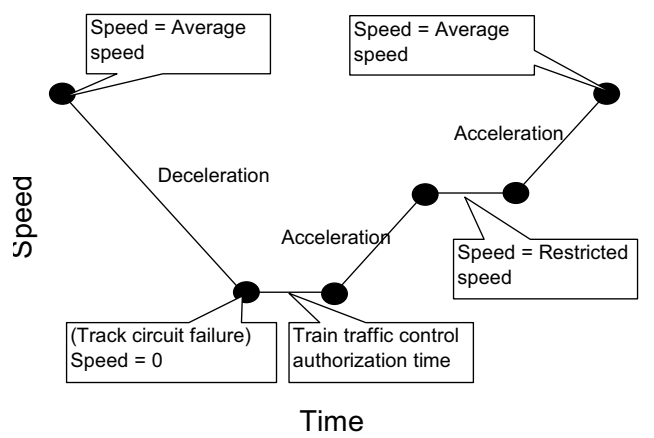

Fig. 10 Explanation of primary delay of trains

When a track circuit fails, the signal turns red and running of a train in the block section concerned is restricted. As shown in the figure, in the case of a track circuit failure, a train decelerates and comes to a standstill just before that particular block. The train then waits for the traffic control room's authorization to proceed on the affected block at a restricted speed. After the train covers that block at a restricted speed, it may accelerate to the original speed. The train is delayed by the extra time taken to cover the distance because of stopping and reducing its speed. If the track circuit is not repaired, then the following trains decelerate and cover that block section at a restricted speed. This adds further primary delay.

Consequently, the total primary delay per year due to the failure of track circuits can be calculated as:

Primary delay $($ minutes/year) $=$ Failure rate of track circuit*Operation hours/year*[Primary delay of train * (Mean down time/Average headway between trains)]

In metro applications, where the headway is small, track circuit failures will give rise to significant secondary delay if the mean down time due to failure is high. The maintenance cost for each of the maintenance policies can be determined by adding the direct maintenance costs and the costs of delay. In the case of condition based maintenance, inspection costs are eliminated but we introduce additional costs for acquisitions of monitoring devices as well as maintenance of monitoring devices. The cost effectiveness of a maintenance policy can be determined by taking a ratio of the availability and the maintenance cost. The higher the costeffectiveness, the better is the maintenance policy.

\section{CONCLUSIONS}

In this article, models for estimation of availability for scheduled maintenance and condition based maintenance have been developed. It has been demonstrated how the availability of the track circuit is influenced by changing the maintenance interval of the track circuit. The article also makes a comparison of scheduled maintenance and condition based maintenance 
based on achieving the availability of track circuit. A framework is presented for the cost of delay. Further, it is shown that cost-benefit analysis of maintenance policies can be carried out to evaluate the cost-effectiveness of each maintenance policy. Cost-effectiveness analysis will yield quantitative results to aid the decision maker with risk analysis, and provide a useful decision tool. Better cost-effectiveness of a system is achieved by higher operational availability and lower maintenance costs during the life cycle of the system.

\section{ACKNOWLEDGEMENT}

The work presented in this paper has been financially supported by the Swedish National Rail Administration (Banverket). The authors would like to acknowledge this support.

\section{REFERENCES}

1 Chen, J., Roberts, C., and Weston, P. Fault detection and diagnosis for railway track circuits using neuro-fuzzy systems, Control Engineering Practice, 2008, 16, 585-596.

2 Niska, S. Measurements and Analysis of Electromagnetic Interferences in the Swedish Railway Systems, PhD Thesis, Division of Operation and Maintenance Engineering, Luleå University of Technology, Luleå, Sweden, vol.76, 2008

3 EN 50126 The Specification and Demonstration of Reliability, Availability, Maintainability and Safety (RAMS) for Railways Applications, Comité Européen de Normalisation Electrotechnique (CENELEC), Brussels, Belgium, 1999.

4 Roberts, C., Dassanayake, H. P. B., Lehrasab, N., and Goodman, C. J. Distributed quantitative and qualitative fault diagnosis: railway junction case study. Control Engineering Practice, 2002, 10(4), 419-429.

5 Murata, T. Petri Nets: Properties, Analysis and Applications. Proceedings of the IEEE, 1989, 77(4), $541-580$.

6 Nyström, B. Use of Availability Concepts in the Railway System, International Journal of Performability Engineering, 2009, 5(2), 103-118 